\title{
Photoluminescent Mechanism of a Proton-transfer Laser Dye in Highly Doped Polymer Films
}

Ken-ichi Sakai ${ }^{*}$, Musubu Ichikawa, Yoshio Taniguchi

Faculty of Textile Science and Technology, Shinshu University, Tokita, Ueda, Nagano 386-8567, Japan

Published in Chem. Phys. Lett. 420(4-6): 405-409 (2006)

${ }^{*}$ Corresponding author. Fax +81 268215811

E-mail address: ksakai@giptc.shinshu-u.ac.jp (K. Sakai). 


\begin{abstract}
Photoluminescent properties in thin films of the proton-transfer (PT) laser dye, 2-(2-hydroxyphenyl)benzothiazole (HBT) were investigated, when it was doped into hole-transport polymer, poly( $N$-vinylcarbazole) and when it was codoped with hole-transport small molecule, $N$, $N^{\prime}$-di(m-tolyl)- $N, N^{\prime}$-diphenylbenzidine (TPD) into polystyrene. The more the doping concentration of HBT was raised up to about $40 \mathrm{wt} \%$, the more its photoluminescent intensity was enhanced without showing excimer or exciplex emissions. The mechanism for such phenomena was discussed in connection with the excited-state intramolecular proton-transfer reaction of HBT.
\end{abstract}

\title{
1. Introduction
}

Organic thin films made of fluorescent small molecules or conjugated polymers are attractive candidates as a gain medium for solid-state lasers, since the rich variety of molecular design makes possible to oscillate at a wide range of wavelengths. With such the advantage, the recent remarkable progress of developing organic light-emitting diodes (OLEDs) encourages the expectation for electrically pumped lasing from organic thin films, i.e., organic laser diodes (OLDs) [1-3]. However, it has been pointed out that there is a serious obstacle to realizing OLDs: the charged carrier (i.e., polarons)-induced absorption loss problem [4,5]. This refers to that within OLED devices, inherent low carrier mobility of organics brings about the situation that the density of polarons is much higher than that of emissive excitons, thereby causing a large absorption loss of stimulated emission by polarons. Also taking into account absorption loss by metal electrode [6-8], it seems to be a reasonable consequence that in OLED structures, gain of emissive medium $(\mathrm{g})$ cannot become predominant over the sum of all such the losses $(\alpha)$, not causing stimulated emission. Toward realization of OLDs, therefore, one should at least tackle two contrastive subjects to make 
net gain positive $(g-\alpha>0)$ : one is the creation of novel laser dyes to raise gain much further, and the other is the drastic innovation for the structure and operation of OLEDs to make losses as small as possible [9-11].

For the former subject, higher gain is expected by increasing number density of laser dye. However, laser dyes under the condition of high concentration often result in causing luminescence quenching by bimolecular annihilation or excimer formation; hence practically in host-guest type of OLED emitting layers, the guest (i.e., laser dye) is used in the concentration diluted below several wt\% [12]. Since such treatment does not provide a way out of overcoming the polaron-absorption problem, it is quite important for developing OLDs to create the laser dyes that do not cause quenching even under high concentration. Of particular notice here is that although chemical modification for preventing intermolecular interactions is effective to avoid quenching [13,14], it will be not favorable to the materials for electroluminescence devices where intermolecular carrier transport is needed.

As a candidate for the laser dye that could be satisfied with such the request, we have been focusing on proton-transfer (PT) laser dyes [15]. Differing from conventional laser dyes, a characteristic property of PT dyes is that the four-level laser scheme for forming a population inversion state is constructed on the basis of tautomerization by excited-state intramolecular proton transfer (ESIPT) [16-19]. From the photopumping measurements for the dilute thin films of the representative PT dye, 2-(2-hydroxyphenyl)benzothiazole (HBT), it was revealed that HBT exhibited better amplified spontaneous emission (ASE) performance, especially at the high doping level beyond $20 \mathrm{wt} \%$ [15]. Although it has no bulky groups acting as an obstacle to intermolecular contacts, concentration quenching rarely occurs, which might give clue to the development of high gain media applicable to the OLD active layers. Moreover, since the wide range of Stokes shift in PT dyes (6000-10000 $\mathrm{cm}^{-1}$ ) can be controlled by chemical modification [20,21], they might have the potential for lowering polaron-absorption loss by finely tuning gain wavelength so as not to 
overlap with polaron-absorption wavelength regions.

With these reasons, we have considered that the PT dyes, such as HBT, are promising for developing OLD. In this work, photoluminescent properties of HBT were investigated, when it was highly doped into hole-transport polymer poly( $N$-vinylcarbazole) (PVK) and when it was highly codoped with hole-transport small molecule $N, N^{\prime}$-di(m-tolyl)- $N, N^{\prime}$-diphenylbenzidine (TPD) into polystyrene (PS). From the consideration for the photoluminescent mechanism, the possibility of HBT as an OLD gain material was discussed.

\section{Experimental}

The chemical structures of the materials used in this study are shown in Chart 1 . They were purchased from Tokyo Kasei Kogyo Co. Ltd., and used without further purification. Dye-doped polymer films were prepared by spin coating from dichloromethane solution onto quartz substrates. Doping concentration was raised up until transparency of the film could be confirmed with naked eyes. The absorption and the PL spectra of the films were recorded on a SHIMAZU UV-3150 UV-Vis-NIR spectrophotometer and on a JASCO FP-750 spectrofluorometer, respectively.

\section{Results and discussion}

The PL spectrum of the 5 wt\% HBT-doped PVK film is shown in Fig. 1, as the solid line on the right-hand side. For excitation, light with wavelength of $340 \mathrm{~nm}$ is used, by which both HBT and PVK can be excited. This spectral shape and position are completely consistent with those of when HBT is doped into electrically inert PS, and thus the emission from PVK, as shown in the dotted line on the right-hand side, is not observed. It is therefore apparent that the radiative decay process of PVK is suppressed by interacting with HBT. The Förster energy transfer from the PVK hosts to the HBT guests is, however, not really expected, since the absorption spectrum of HBT (Fig. 1, solid line, left-hand side) is rather located at the higher energy side than the PL spectrum of PVK, 
and the spectral overlap between them is quite poor. In fact, on the basis of the spectral overlap integral, the Förster critical radius $R_{0}$ is calculated to be $14 \AA$ by using the following formula [22]:

$$
R_{0}^{6}=\frac{9000 \ln 10 \kappa^{2} \Phi_{D}}{128 \pi^{5} n^{4} N_{A}} \int \frac{F_{D}(v) \varepsilon_{A}(v)}{v^{4}} d v
$$

where $\kappa^{2}$ is an orientation factor (2/3 for random orientation), $\Phi_{\mathrm{D}}$ and $n$ are the PL quantum yield and the refractive index of the host PVK, respectively, $\left(\Phi_{\mathrm{D}}=0.1, n=1.5\right)$ [23], $N_{A}$ is Avogadro's number, and $F_{\mathrm{D}}$ and $\varepsilon_{\mathrm{A}}$ are the normalized PL spectrum of PVK and the molar extinction coefficient spectrum of HBT, respectively, both expressed as a function of energy in wavenumber $(v)$. The calculated $R_{0}$ value is quite small, if compared to that in the MEH-PPV/porphyrin system $\left(R_{0}=25\right.$ A) [24], which is relatively small among the values in the Förster type of organic films reported thus far. Smaller $R_{0}$ indicates that the average host-guest distance must become closer by increasing guest concentration to achieve complete energy transfer (i.e., complete quenching of the host emission). Practically in the MEH-PPV/porphyrin system, 9-10 wt\% doping of porphyrin is needed to quench the MEH-PPV emission. However, in the PVK/HBT system, the PVK emission is disappeared, even at the HBT doping level of 5 wt\%, indicating deviation from the Förster energy-transfer model. It was impossible to compare the PL efficiency of the films between when doped into PVK and when into PS, since the amount of HBT within the PVK film could not be exactly estimated due to the absorption spectral overlap between HBT and PVK.

Figure 2a shows the HBT concentration dependence of PL spectra in the HBT/PVK system. Actually, with a small HBT doping amount of $0.5 \mathrm{wt} \%$, the PVK emission in the range 350-500 nm is strongly quenched, and becomes not detectable beyond $2 \mathrm{wt} \%$. This surely implies that HBT acts as an effective trap for excitons formed on PVK. It should be also noticed that with further increasing the doping concentration, the HBT emission intensity is increased up to $40 \mathrm{wt} \%$, leaving the spectral profile and the location unchanged. Compared with conventional laser dyes, so much less of the concentration quenching in HBT could provide a possibility of forming higher gain 
media. In view of ASE threshold energy, the lowest was obtained at $26 \mathrm{wt} \%$ [15], beyond which the crystallinity of HBT might reduce morphological homogeneity within the film, thereby causing a scattering loss.

Similar doping concentration dependence of PL spectra is also seen in HBT/TPD blended PS films. Figure 2b shows the PL spectral change at different weight ratio of HBT:TPD. The strong PL intensity of TPD itself is drastically decreased to about one-fiftieth with the HBT addition of 10 wt\%, which corresponds to the molar ratio of HBT:TPD $=1: 6$. Complete quenching of the TPD emission occurs when HBT:TPD = 4:6 (wt\%), being almost equal to 1:1 (mol \%). No spectral overlap between the HBT absorption and the TPD emission (Fig. 1) denies as well the possibility of Förster energy-transfer.

Such anomalous PL behaviors of HBT seem to be related to its emission process based on ESIPT. For the sake of comparison, PL measurements were performed on 2-phenylbenzothiazole (PBT) without an intramolecular hydrogen bond. Figure 3 shows the PL spectra of PBT-doped PS and PVK films with different doping concentrations. In the PS films (circle lines), it is confirmed that the increase of PBT concentration causes the spectral peak shift and broadening to the longer wavelength side. This should result from additional radiative decay processes derived from PBT intermolecular contacts, such as excimer formation. On the other hand, when doped into PVK, redshift of the peak is observed with increase of the doping concentration (rhombic lines). Neither the PL spectrum of PBT nor of PVK coincides with that of the PBT-doped PVK film, although PVK emission still remains for the $5 \mathrm{wt} \%$ PBT-doped PVK film (white rhombic line). This suggests that PBT forms an exciplex with PVK. Similar results were also obtained for the PBT/TPD blend system (data not shown); in fact, it is known that blends of blue-emitting molecules often give rise to exciplex emission [25].

Since the molecular structure of HBT is almost the same as that of PBT, it could be safely said that the ground and excited states of HBT before ESIPT (i.e., enol form) resemble those of PBT. 
Therefore, it seems valid to assume that HBT also forms an exciplex with PVK or TPD, although its emission is not observed. Actually, such the assumption provides a rational explanation for the anomalous PL behaviors of HBT; conversely, in the HBT/PVK and the HBT/TPD blend systems, where Förster energy transfer is not expected, strong quenching phenomena of PVK and TPD cannot be explained as long as the exciplex formation with HBT is assumed. Figures $4 \mathrm{a}$ and $4 \mathrm{~b}$ show the orbital interaction and the potential energy curve diagrams expressing the mechanism of exciplex formation, respectively. An exciplex state $\left[D^{*} A\right]$ is constructed by interaction between the excited state of donor $\left(D^{*}\right)$ and the ground state of acceptor $(A)$, in which orbital mixing between the lowest unoccupied molecular orbitals (LUMOs) of $D$ and A plays a significant role (Fig. 4a) [25]. If there is no such orbital interaction between $D^{*}$ and $A$, emissions from both species should be observable. However, if exciplexes are formed, original emissions from $D$ and $A$ become weaker or vanished, and at the same time emission depicted in Fig. 4b newly appears. On the basis of these descriptions for exciplex formation, the potential energy curves when HBT acts as $A$ are drawn as Fig. 4c. In this case, the enol structure $\left(A_{e}\right)$ of HBT, which is in the minimum on the ground state, interacts with $D^{*}$ to form a $\left[D^{*} A_{e}\right]$. Emission from $\left[D^{*} A_{e}\right]$ is, however, not occurred because of the transition to the excited state of keto structure $\left(A_{k}{ }^{*}\right)$ via ESIPT. Reflecting the large Stokes shift of HBT (9000 $\mathrm{cm}^{-1}$ ), there will be a large orbital energy difference between the LUMOs of $A_{e}$ and $A_{k}$, and eventually between those of $D$ and $A_{k}$. Such a situation should not bring about effective orbital interaction between $D^{*}$ and $A_{k}$ enough to form a $\left[D^{*} A_{k}\right]$. As a result, only observable is the emission from $A_{k}^{*}$ that is the original radiative decay process of HBT with gain.

Finally, we may note that the OLED devices were tentatively fabricated using the HBT/PVK or the HBT/TPD/PS film as emitting layer. Although electroluminescence from HBT has been observed even under the high doping concentration, the device performance such as current efficiency is poor at the present stage. Further improvement and development based on the use of PT dyes are in progress. 


\section{Conclusion}

It is the ESIPT reaction of HBT that is involved in the origin of both the following phenomena: the strong quenching of PVK and TPD by small addition of HBT, and the enhancement of PL intensity even under high HBT concentration. The former results from a $\left[D^{*} A_{e}\right]$ formation and followed by ESIPT, while the latter does from no $\left[D^{*} A_{k}\right]$ formation after ESIPT. Of particular importance here is a large mismatch between LUMO energy levels of $A_{e}$ and $A_{k}$, achieved by ESIPT. This should prevent not only the formation of $\left[D^{*} A_{k}\right]$ exciplex, but also that of $\left[\mathrm{A}_{e}{ }^{*} \mathrm{~A}_{k}\right]$ excimer for which LUMO-LUMO interaction between $\mathrm{A}_{e}{ }^{*}$ and $\mathrm{A}_{k}$ is responsible. The use of PT laser dyes, such as HBT, therefore, opens a way of raising dye concentration (i.e., gain) without affected by intermolecular interactions.

\section{Acknowledgements}

This work was supported by the CLUSTER of Ministry of Education, Culture, Sports, Science and Technology.

\section{References}

[1] M.D. McGehee, A.J. Heeger, Adv. Mater. 12 (2000) 1655.

[2] V.G. Kozlov, G.. Parthasarathy, P.E. Burrows, V.B. Khalfin, J. Wang, S.Y. Chou, S.R. Forrest, IEEE J. Quan. Elect. 36 (2000) 18.

[3] N. Tessler, Adv. Mater. 11 (1999) 363.

[4] V.G. Kozlov, P.E. Burrows, G. Parthasarathy, S.R. Forrest, Appl. Phys. Lett. 74 (1999) 1057.

[5] N. Tessler, D.J. Pinner, V. Cleave, D.S. Thomas, G. Yahioglu, P.Le Barny, R.H. Friend, Appl. Phys. Lett. 74 (1999) 2764. 
[6] M. Reufer, S. Riechel, J.M. Lupton, J. Feldmann, U. Lemmer, D. Schneider, T. Benstem, T. Dobbertin, W. Kowalsky, A. Gombert, K. Forberich, V. Wittwer, U. Scherf, Appl. Phys. Lett. 84 (2004) 3262.

[7] H. Yamamoto, T. Oyamada, H. Sasabe, C. Adachi, Appl. Phys. Lett. 84 (2004) 1401.

[8] M. Reufer, J. Feldmann, P. Rudati, A. Ruhl, D. Müller, K. Meerholz, C. Karnutsch, M. Gerken, U. Lemmer, Appl. Phys. Lett. 86 (2005) 221102.

[9] N. Tessler, D.J. Pinner, V. Cleave, P.K.H. Ho, R.H. Friend, G. Yahioglu, P.Le Barny, J. Gray, M.de Souza, G. Rumbles, Synthetic Metals 115 (2000) 57.

[10] M. Pauchard, J. Swensen, D. Moses, A.J. Heeger, E. Perzon, M.R. Anderson, J. Appl. Phys. 94 (2003) 3543.

[11] K. Sakai, T. Sonoyama, T. Tsuzuki, M. Ichikawa, Y. Taniguchi, Chem. Lett. 34 (2005) 212.

[12] V.G. Kozlov, V. Bulovic, P.E. Burrows, M. Baldo, V.B. Khalfin, G. Parthasarathy, S.R. Forrest, Y. You, M.E. Thompson, J. Appl. Phys. 84 (1998) 4098.

[13] S. Yokoyama, A. Otomo, S. Mashiko, Appl. Phys. Lett. 80 (2002) 7.

[14] S. Kim, S.Y. Park, I. Yoshida, H. Kawai, T. Nagamura, J. Phys. Chem. B 106 (2002) 9291.

[15] K. Sakai, T. Tsuzuki, Y. Itoh, M. Ichikawa, Y. Taniguchi, Appl. Phys. Lett. 86 (2005) 081103.

[16] A.U. Khan, M. Kasha, Proc. Natl. Acad. Sci. U.S.A. 80 (1983) 1767.

[17] P. Chou, D. McMorrow, T.J. Aartsma, M. Kasha, J. Phys. Chem. 88 (1984) 4596.

[18] A.U. Acuña, A. Costela, J.M. Muñoz, J. Phys. Chem. 90 (1986) 2807.

[19] S. Park, O.-H. Kwon, S. Kim, S. Park. M.-G. Choi, M. Cha, S.Y. Park, D.-J. Jang, J. Am. Chem. Soc. 127 (2005) 10070.

[20] D.L. Williams, A. Heller, J. Phys. Chem. 74 (1970) 4473.

[21] A. Costela, F. Amat, J. Catalán, A. Douhal, J.M. Figuera, J.M. Muñoz, A.U. Acuña, Optics Comm. 64 (1987) 457.

[22] T. Förster, Ann. Phys. 2 (1948) 55. 
[23] L. Miozzo, A. Papagni, M. Cerminara, F. Meinardi, R. Tubino, C. Botta, Chem. Phys. Lett. 399 (2004) 152.

[24] J. Morgado, F. Cacialli, R. Iqbal, S.C. Moratti, A.B. Holmes, G. Yahioglu, L.R. Milgrom, R.H. Friend, J. Mater. Chem. 11 (2001) 278.

[25] J. Thompson, R.I.R. Blyth, M. Mazzeo, M. Anni, G. Gigli, R. Cingolani, Appl. Phys. Lett. 79 (2001) 560.

\section{Captions}

Chart 1. Chemical structures of materials used in this study. The enol form of HBT is tautomerized to the keto form by ESIPT.

Fig. 1. Absorption and PL spectra of the materials discussed in this study: absorption of a HBT-doped PS film (solid line, left-hand side), PL of a 5wt\% HBT-doped PVK film (solid line, right-hand side), absorption and PL of a PVK neat film (dotted lines, left- and right-hand side, respectively), and TPD-doped PS film (dashed line). For all PL measurements, excitation wavelength is $340 \mathrm{~nm}$.

Fig. 2. Doping concentration dependences of PL spectra (excitation at $340 \mathrm{~nm}$ ) in (a) HBT-doped PVK films and in (b) HBT/TPD-codoped PS films.

Fig. 3. PL spectra of PBT-doped PS and PVK films at the doping concentrations of $5 \mathrm{wt} \%$ and 26wt\% (excitation at $300 \mathrm{~nm})$.

Fig. 4. Schematic diagrams of the exciplex formation mechanism described by (a) orbital mixing and by (b) crossing of potential energy curves. (c) is the case where PT dyes such as HBT act as acceptor. The exciplex emission from $\left[D^{*} A_{e}\right]$ is suppressed by ESIPT. Exciplex between $D$ and $A_{k}$ is not formed (i.e., their potential energy curves of the excited states are not crossing) due to the large mismatch of their LUMO energy levels. 

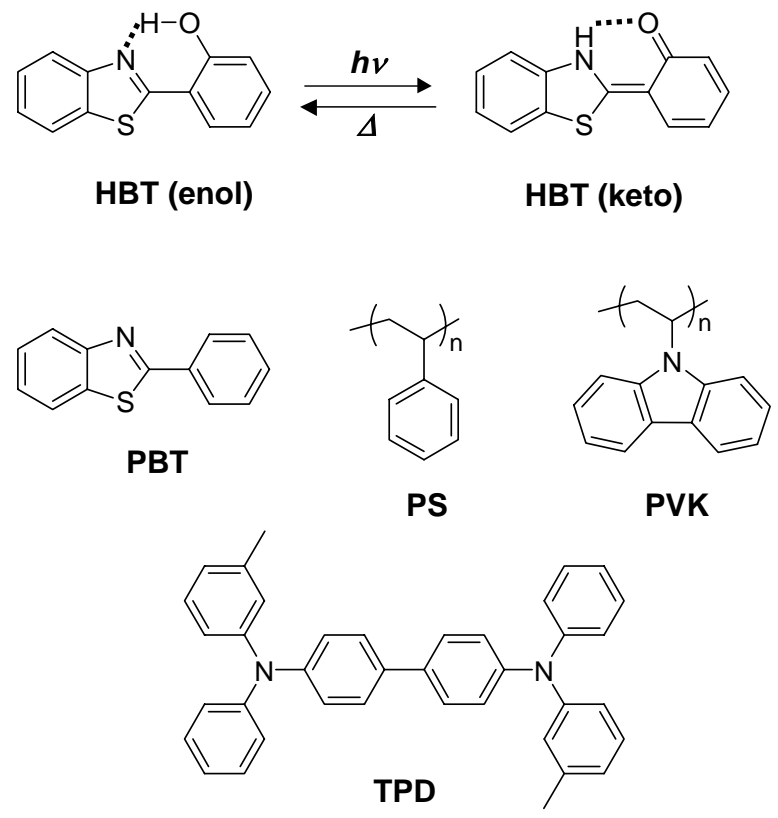

Chart 1 


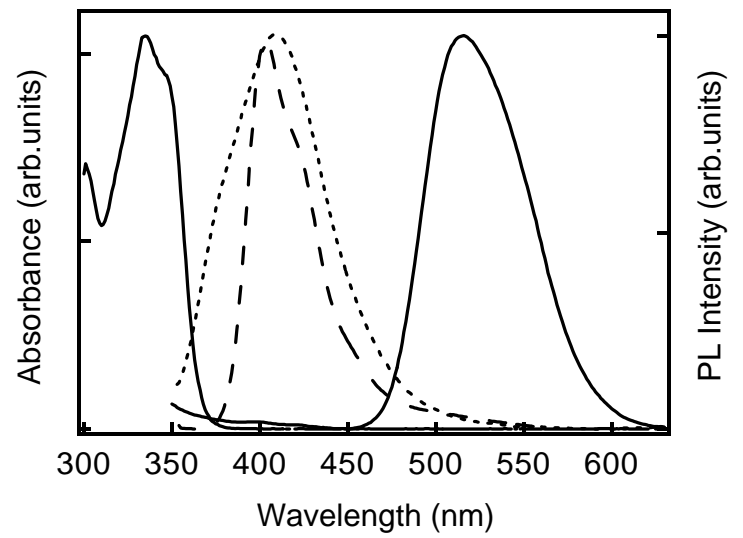

Fig. 1 

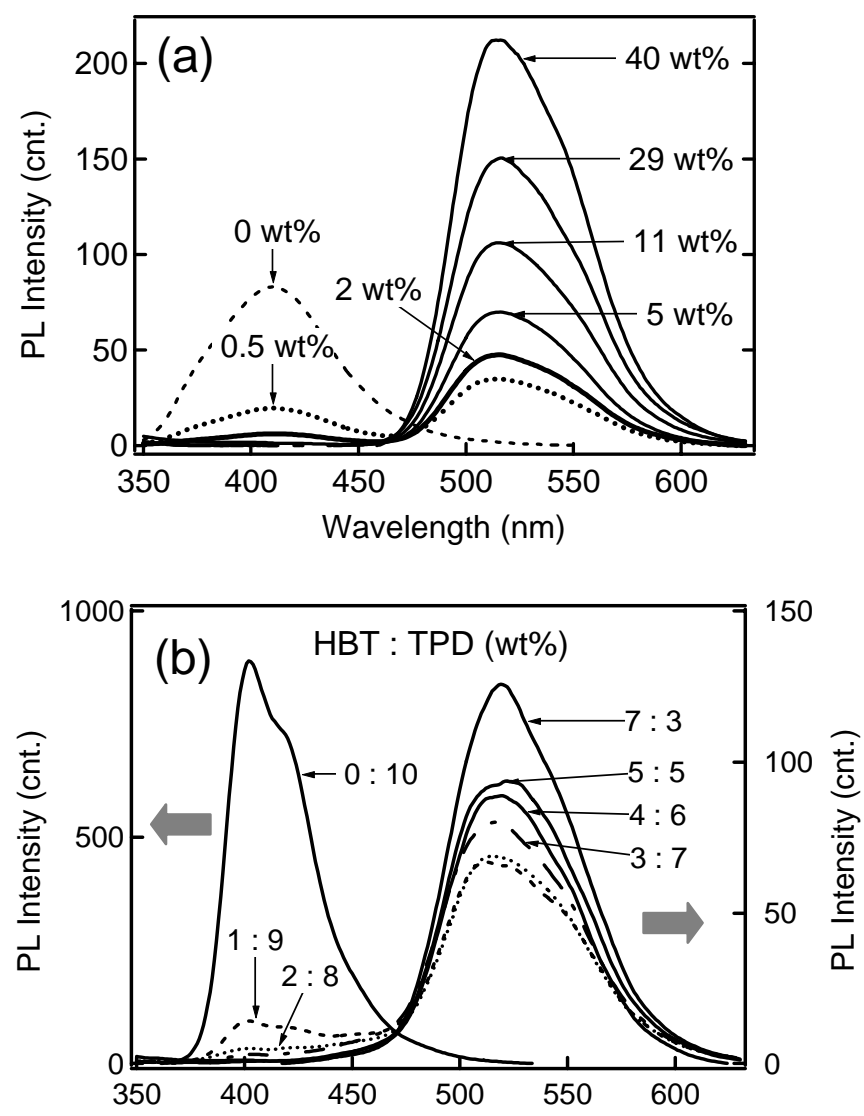

Fig. 2 


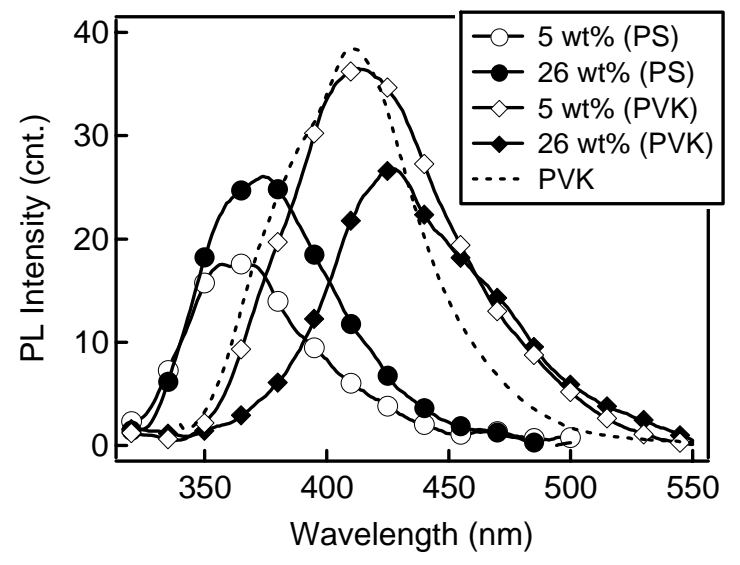

Fig. 3 

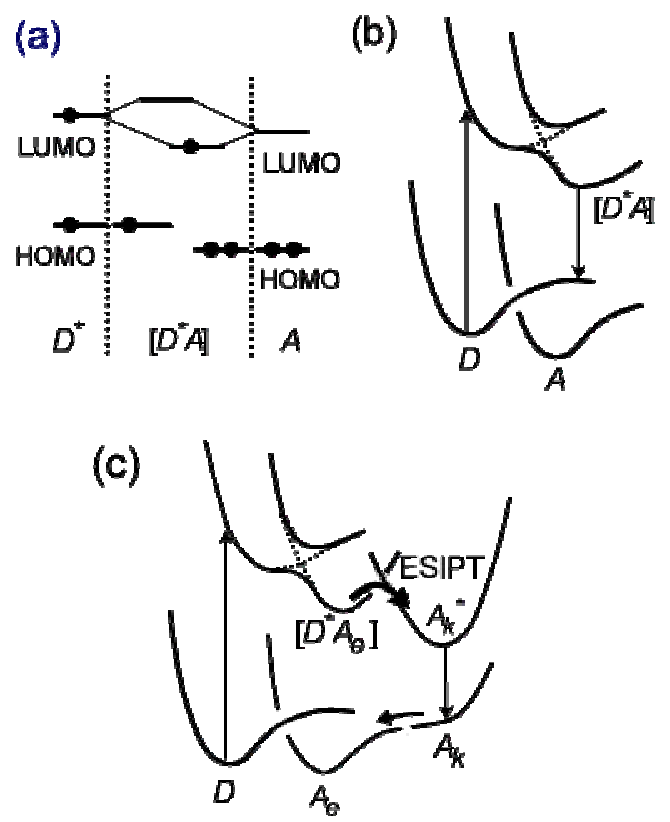

Fig. 4 